\title{
Tanshinone IIA suppresses gastric cancer cell proliferation and migration by downregulation of FOXM1
}

\author{
JIAO YU ${ }^{1 *}$, XIAOXIA WANG ${ }^{2 *}$, YUHUA LI $^{1}$ and BIN TANG ${ }^{3}$ \\ ${ }^{1}$ Linyi Hospital of Traditional Chinese Medicine, Linyi, Shandong 276000; ${ }^{2}$ Linyi Tumor Hospital, Linyi, Shandong 276000; \\ ${ }^{3}$ Lanzhou Hengdao Chinese Medicine Institute, Lanzhou, Shandong 730000, P.R. China
}

Received July 27, 2016; Accepted September 9, 2016

DOI: $10.3892 /$ or.2017.5408

\begin{abstract}
Tanshinone IIA (TSN) exhibits a variety of anticancer effects. However, whether it inhibits gastric cancer (GC) cell proliferation and migration and the mechanism remain unclear. In the present study, different concentrations of TSN were co-incubated with SGC-7901 cells. The pcDNA-FOXM1 or FOXM1-siRNA plasmid was transfected into cells before treatment with $5 \mu \mathrm{g} / \mathrm{l} \mathrm{TSN}$. The proliferation and migration abilities of the SGC-7901 cells were tested by MTT and wound healing assays. Western blotting was used to investigate the expression levels of P21, Ki-67, PCNA, MMP-2, MMP-9 and FOXM1. We found that compared with the control, the proliferation and migration abilities of the SGC-7901 cells were decreased after incubation with different concentrations of TSN in a dose-dependent manner $(\mathrm{p}<0.01)$. Moreover, the expression levels of Ki-67, PCAN, MMP-2, MMP-9 and FOXM1 were decreased, and P21 was increased in the TSN-treated SGC-7901 cells $(\mathrm{p}<0.01)$. In addition, downregulation of FOXM1 by FOXM1-siRNA had the same effect as TSN on SGC-7901 cells, and overexpression of FOXM1 partly abrogated TSN-mediated inhibition of SGC-7901 cell proliferation and migration. These results suggested that TSN inhibits SGC-7901 cell proliferation and migration by downregulation of FOXM1.
\end{abstract}

\section{Introduction}

Gastric cancer (GC) is the fourth most frequently occurring malignant tumor, and exhibits considerable geographic

Correspondence to: Dr Jiao Yu, Linyi Hospital of Traditional Chinese Medicine, Linyi, Shandong 276000, P.R. China

E-mail: yuyuguosh@sina.com

Dr Xiaoxia Wang, Linyi Tumor Hospital, Linyi, Shandong 276000, P.R. China

E-mail: xiaoxia.wang@hotmail.com

${ }^{*}$ Contributed equally

Key words: gastric cancer, tanshinone IIA, FOXM1, proliferation, migration variation. GC ranks as the second leading cause of cancer-related deaths worldwide. Environmental factors and the accumulation of genetic alterations are currently thought to be the leading causes of GC. Benefiting from advances in the early diagnosis and adjuvant treatment of GC, the 5-year disease-free survival rate of patients has increased in the last few decades. However, many patients who are treated with such therapies still experience disease progression (1). Thus, new treatment choices are critically required.

Tanshinone IIA (TSN), a commonly used Chinese traditional drug for the treatment of cardiovascular and cerebrovascular diseases such as atherosclerosis, angina pectoris and acute ischemic stroke $(2,3)$, has also exhibited a variety of anticancer effects since Wang et al first reported that TSN suppressed breast cancer progression by inhibiting the proliferation and by inducing apoptosis of cancer cells (4). Subsequent research further confirmed the anticancer effect of TNS on esophageal, prostate, colorectal, lung and GC (5-8). However, the mechanism by which TSN suppresses GC progression remains unclear.

The forkhead box M1 (FOXM1) gene is a member of the FOX family, and has been shown to play important roles in cell fate decisions. In tumor genesis, numerous studies have shown that FOXM1 is significantly increased in multiple human cancers such as esophageal and breast cancer, hepatocellular carcinoma, colorectal cancer and GC (9-12). In addition, its overexpression is closely correlated with tumor progression and metastasis (11) and downregulation of FOXM1 inhibits tumor progression. However, the function of FOXM1 in TSN-induced inhibition of gastric tumor metastasis has not been reported.

In the present study, we demonstrated that TSN inhibits the proliferation and migration of GC cells, and also demonstrated that downregulation of FOXM1 is the key underlying mechanism.

\section{Materials and methods}

Cell culture and transfection. The human GC cell line (SGC-7901) was obtained from the American Type Culture Collection (ATCC; Rockville, MD, USA) and kept in RPMI-1640 medium with 10\% fetal bovine serum (FBS) (Gibco, Carlsbad, CA, USA), $1 \%$ of $100 \mathrm{U} / \mathrm{ml}$ penicillin and $1 \%$ of $100 \mathrm{mg} / \mathrm{ml}$ streptomycin sulfates. The cells were incubated in humidified incubators with $5 \% \mathrm{CO}_{2}$ at $37^{\circ} \mathrm{C}$. 
Human FOXM1 gene or FOXM1-siRNA was constructed into the pcDNA3.1+HA vector by Life Technologies (GeneChem, Shanghai, China), and the empty vector served as the negative control. For transfection, after the cells were cultured to 70-80\% confluency, pcDNA3.1+HA-FOXM1, pcDNA3.1+HA empty vector, pcDNA3.1+FOXM1-siRNA or pcDNA3.1+NC-siNRA was transfected using Lipofectamine 2000 (Invitrogen, Carlsbad, CA, USA) according to the manufacturer's instructions.

Quantitative real-time PCR. Total RNA was extracted from tissues and cells using TRIzol reagent (Invitrogen), and then miRNAs were reverse-transcribed to cDNA using a reverse transcription kit (Takara, Tokyo, Japan). Quantitative real-time PCR (qRT-PCR) was performed using SYBR-Green PCR kit on an ABI 7500 Fast Real-Time PCR system according to the manufacturer's instructions. The expression of FOXM1 mRNA was normalized to U6. All experiments were carried out in triplicate. The $2^{-\Delta \Delta C t}$ calculation method was used to calculate the relative expression of the genes.

Cell proliferation assay. The proliferation ability of SGC-7901 cells was assessed by the 3-(4,5-dimethylthiazol-2-yl)-2,5-diphenyltetrazolium bromide (MTT; Sigma-Aldrich, St. Louis, MO, USA) assay. Approximately $10^{4}$ cells were seeded into each well of 96-well plates. Then, SGC-7901 cells were transfected with pcDNA3.1+HA-FOXM1, pcDNA3.1+HA empty vector, pcDNA3.1+FOXM1-siRNA or pcDNA3.1+NC-siNRA following the manufacturer's instructions. After $6 \mathrm{~h}$ of transfection, the cells were treated with TSN or a placebo. After 12, 24 or $48 \mathrm{~h}$ of incubation, $25 \mu \mathrm{l}$ of MTT $(5 \mathrm{mg} / \mathrm{ml})$ was added to each well and the plates were incubated for $4 \mathrm{~h}$ at $37^{\circ} \mathrm{C}$. Then, the precipitates in each well were solubilized with $150 \mu \mathrm{l}$ of dimethyl sulfoxide (DMSO; Sigma-Aldrich), and the plates were read on a microplate reader (Anthos Labtec Instruments, Salzburg, Austria) at $490 \mathrm{~nm}$. Values were normalized using the control value.

Cell migration assay. The migration ability of the SGC-7901 cells was measured by a wound healing assay. Approximately $10^{6}$ cells were seeded into each well of 6 -well plates. Then, SGC-7901 cells were transfected with pcDNA3.1+HA-FOXM1, pcDNA3.1+HA empty vector, pcDNA3.1+FOXM1-siRNA or pcDNA3.1+NC-siNRA following the manufacturer's instructions. After $6 \mathrm{~h}$ of transfection, a cell scratch spatula was used to scratch the cell layer when cells reached $\sim 90 \%$ confluency. After being washed with warm phosphate-buffered saline (PBS) 3 times, the cells were treated with TSN or a placebo. Then, the cells continued to be incubated at $37^{\circ} \mathrm{C}$ for $18 \mathrm{~h}$. A digital camera system (Olympus Corp., Tokyo, Japan) was used to acquire images of the scratches of the cells after incubation at 0 and $18 \mathrm{~h}$.

Western blotting. Cells after treatment, were extracted with RIPA lysis buffer (Biyuntian, Hangzhou, China). Protein lysates were then separated by $10 \%$ sodium dodecyl sulfate-polyacrylamide gel electrophoresis (SDS-PAGE) and transferred to polyvinylidene fluoride (PVDF) membranes (Millipore, Billerica, MA, USA). After blocking with $5 \%$ non-fat milk for $2 \mathrm{~h}$ at room temperature, the membranes were incubated with the primary antibodies: P21 (1:1,000), Ki-67 (1:1,000), PCNA (1:1,000), MMP-2 (1:1,000), MMP-9 (1:2,000), FOXM1 $(1: 1,000)$ and $\beta$-actin $(1: 1,000)$ (all from Abcam, Cambridge, MA, USA) overnight at $4^{\circ} \mathrm{C}$. Then, the membranes were incubated in HRP-linked secondary antibodies (Santa Cruz Biotechnology, Santa Cruz, CA, USA) for 2 h. Western blotting signals were detected using the ECL Plus kit (Biyuntian). Each experiment was repeated 3 times, independently.

Statistical analysis. All statistical analyses were performed using SPSS 20.0. Data are presented as the mean \pm SD. Differences between groups were analyzed using Student's t-test or one-way ANOVA analysis. All experiments were repeated at least 3 times. The value of $\mathrm{p}<0.05$ was considered to indicate a statistically significant result.

\section{Results}

TSN inhibits SGC-7901 cell proliferation. An MTT assay was performed to test the effect of TSN on SGC-7901 cell proliferation. As shown in Fig. 1A, TSN markedly inhibited the proliferation ability of SGC-7901 cells in a dose-dependent manner $(\mathrm{p}<0.05)$. Moreover, we also found that TSN increased the expression of P21 and decreased the expression levels of PCNA and Ki-67 in the SGC-7901 cells in a dose-dependent manner (Fig. 1B; p<0.05). These results indicated that TSN inhibited SGC-7901 cell proliferation.

TSN suppresses SGC-7901 cell migration. Cells were incubated with increasing concentrations of TSN. Images of the scratches were captured at 0 and $18 \mathrm{~h}$ after TSN was added. We found that TSN markedly inhibited SGC-7901 cell migration after $18 \mathrm{~h}$ (Fig. 2A; p<0.01). In agreement, the results from western blot analysis showed that TSN also suppressed the expression of MMP-2 and MMP-9 (Fig. 2B). These results indicated that TSN inhibited SGC-7901 cell migration.

TSN decreases the expression of FOXM1 in SGC-7901 cells. After SGC-7901 cells were incubated with increasing concentrations of TSN for $48 \mathrm{~h}$, western blot analysis showed that the expression of FOXM1 was significantly decreased by TSN in a dose-dependent manner (Fig. 2B; p<0.01). Considering the critical role of FOXM1 in the development of cancer, these results prompted us to focus on FOXM1 in the following experiment.

Downregulation of FOXM1 inhibits SGC-7901 cell proliferation and migration similarly to TSN. FOXM1-siRNA was used to knock out the expression of FOXM1 in the SGC-7901 cells. Fluorescence showed that the plasmid was successfully transfected into the SGC-7901 cells (Fig. 3A). Western blot analysis showed that the protein levels of FOXM1 were decreased in the cells transfected with pcDNA3.1+FOXM1-siRNA compared with the NC-siRNA (Fig. 3B; p<0.01). Moreover, quantitative real-time PCR demonstrated similar results that were obtained from the western blot analysis (Fig. 3C; p<0.01).

In a similar manner to TSN $(5 \mu \mathrm{g} / \mathrm{l})$, downregulation of FOXM1 by FOXM1-siRNA also suppressed SGC-7901 proliferation (Fig. 4A; p<0.05). In agreement, western blot analysis revealed that similarly to TSN, downregulation of FOXM1 

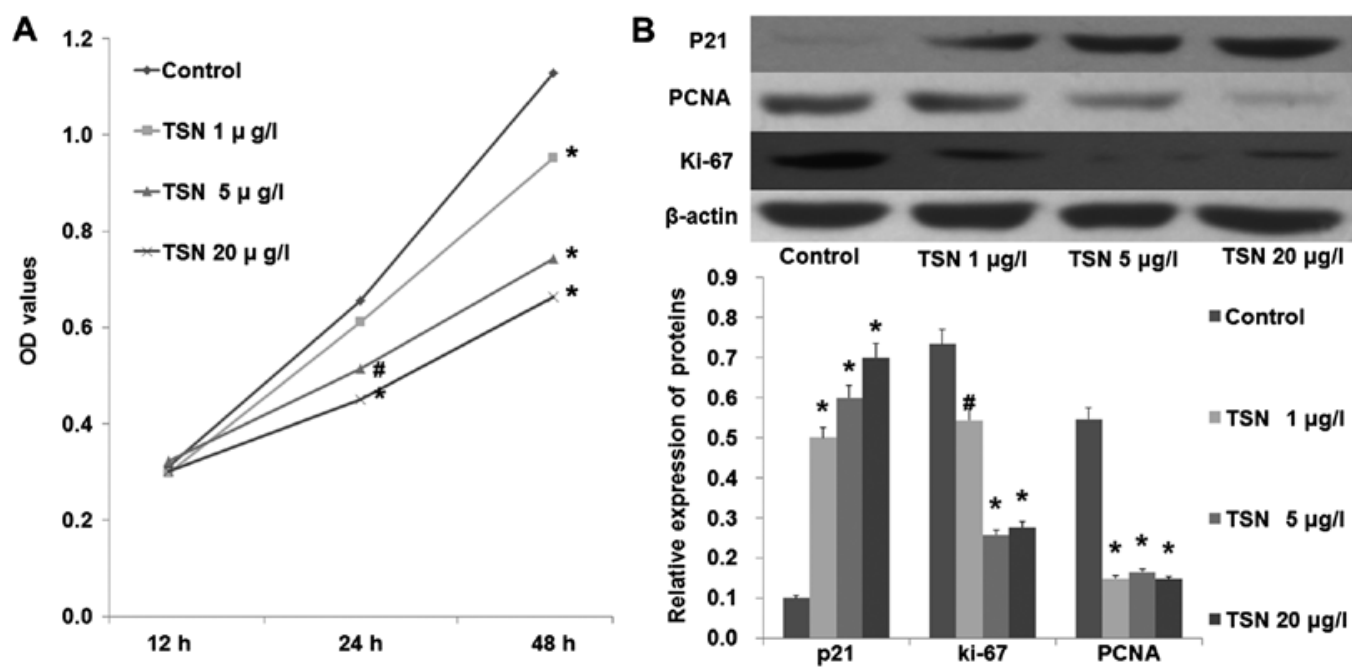

Figure 1. TSN inhibits SGC-7901 cell proliferation. (A) MTT assay demonstrated that TSN inhibited SGC-7901 proliferation in a dose-dependent manner. (B) TSN increased the expression of P21 and decreased the expression of PCNA and Ki-67 in a dose-dependent manner; ${ }^{*} \mathrm{p}<0.05$ compared with the control, " $\mathrm{p}<0.01$ compared with the control. TSN, tanshinone IIA.

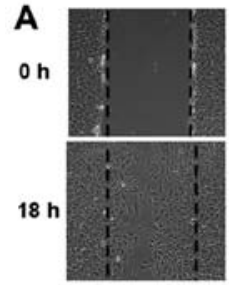

Control

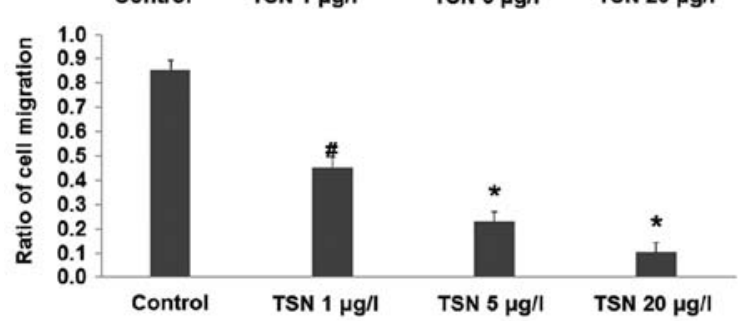

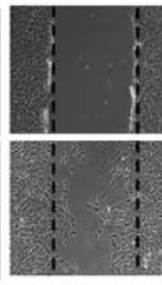

TSN $1 \mu g / l$

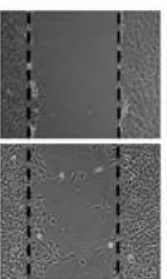

TSN $5 \mu g / l$

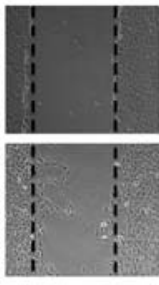

TSN $20 \mu g / 1$

SN $20 \mu g / l$
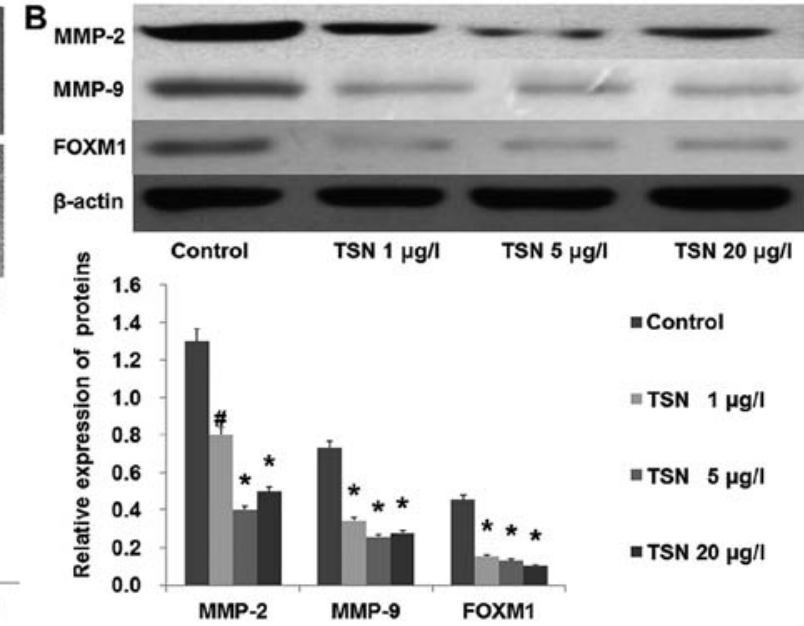

- Control

$=\mathrm{TSN} 1 \mu \mathrm{g} / \mathrm{l}$

- TSN $5 \mu g / l$

= TSN $20 \mu g /$

Figure 2. TSN inhibits SGC-7901 cell migration. (A) Wound healing assay demonstrated that TSN inhibited SGC-7901 cell migration in a dose-dependent manner. (B) TSN decreased the expression of MMP-2, MMP-9 and FOXM1 in a dose-dependent manner; ${ }^{*} \mathrm{p}<0.05$ compared with control, ${ }^{*} \mathrm{p}<0.01$ compared with the control. TSN, tanshinone IIA.

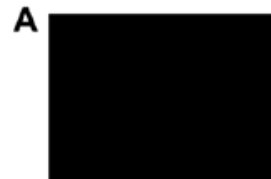

C
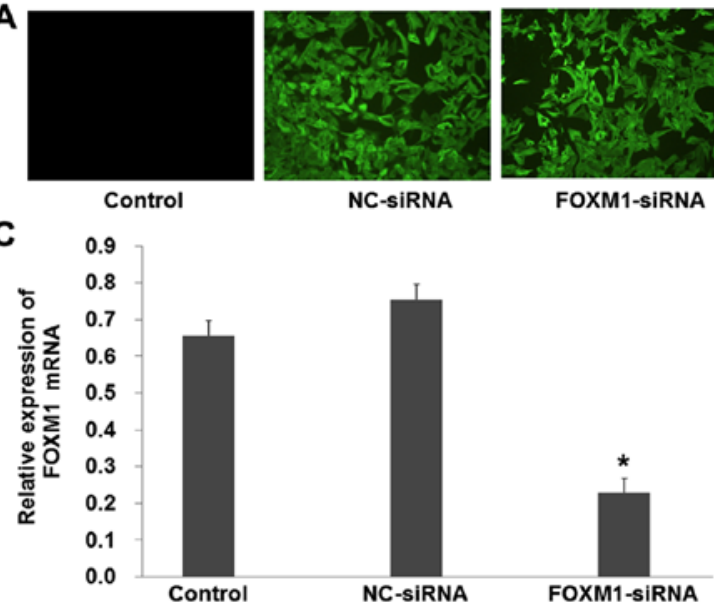

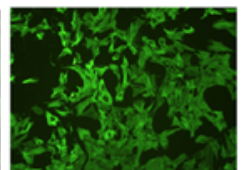

B $_{\text {FoxM1 }}$
$\beta$-actin

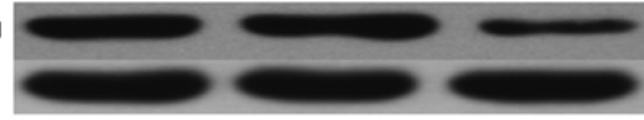

Control

NC-siRNA

FOXM1-sIRNA

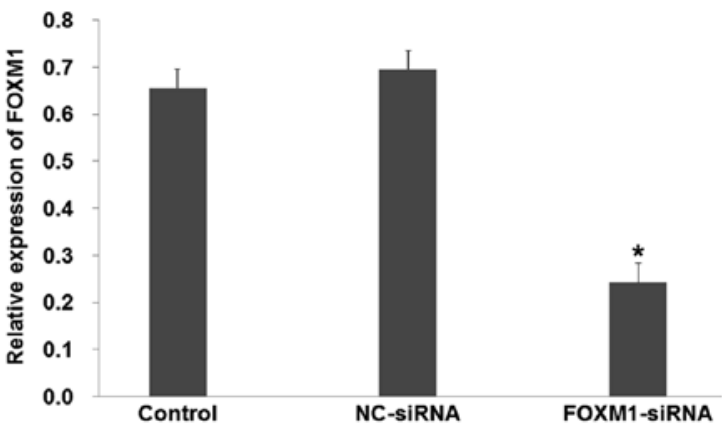

Figure 3. Downregulation of FOXM1 in SGC-7901 cells. (A) Fluorescence showed that the plasmid was successfully transfected into the SGC-7901 cells (B) Western blot analysis demonstrated that the expression of FOXM1 was decreased in the cells transfected with FOXM1-siRNA. (C) RT-PCR showed that the expression of FOXM1 mRNA was decreased in the cells transfected with FOXM1-siRNA; ${ }^{*}$ p $<0.01$ compared with NC-siRNA. 

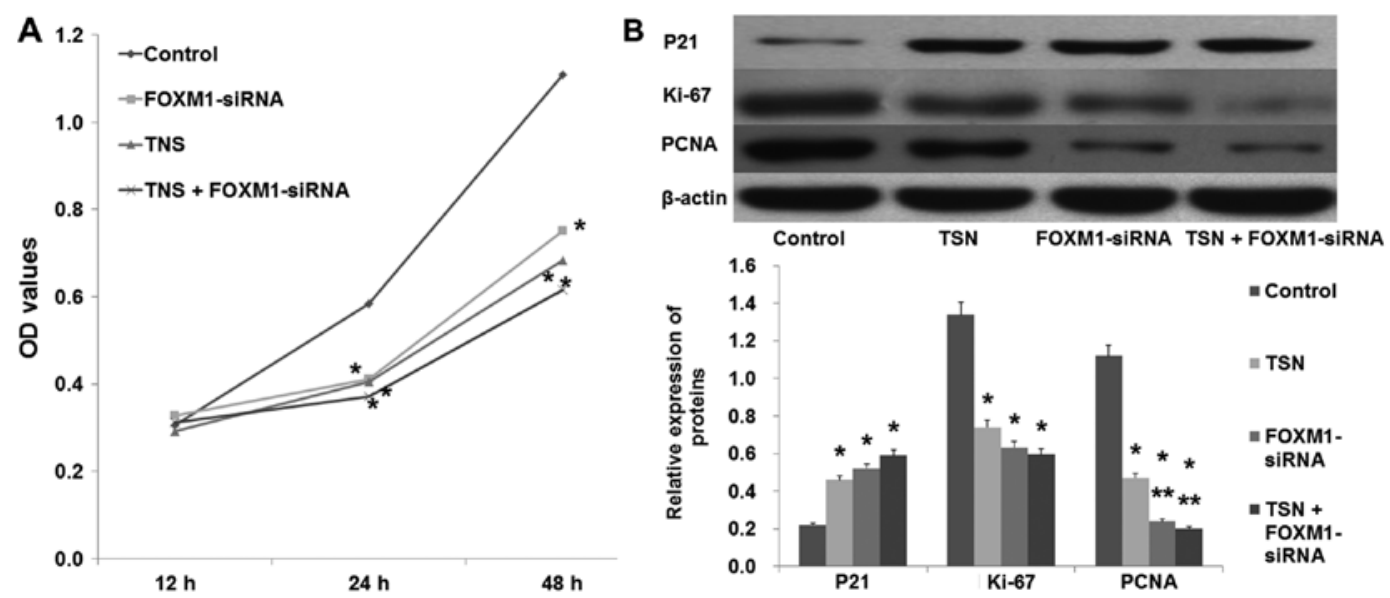

Figure 4. Downregulation of FOXM1 inhibits SGC-7901 cell proliferation. (A) An MTT assay showed that downregulation of FOXM1 inhibited SGC-7901 cell proliferation. (B) Downregulation of FOXM1 increased the expression of P21 and decreased the expression levels of Ki-67 and PCNA; ${ }^{*}$ p $<0.01$ compared with the control; ${ }^{* *} \mathrm{p}<0.01$ compared with TSN. TSN, tanshinone IIA.
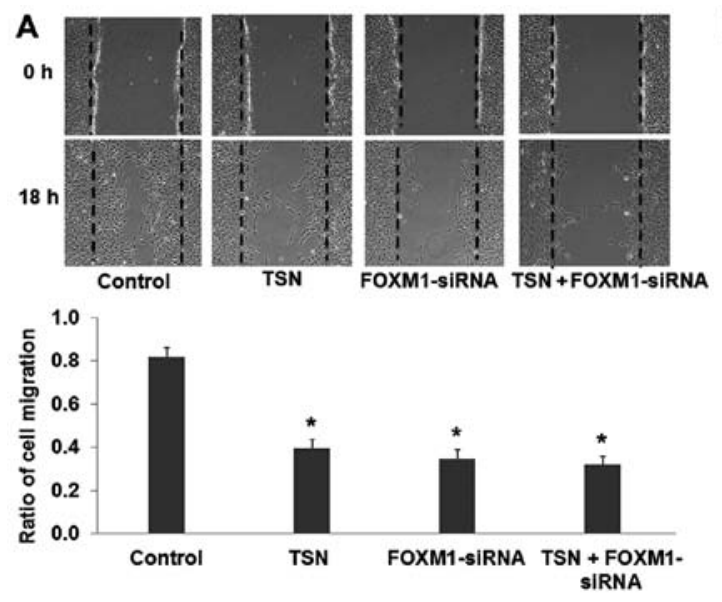

B
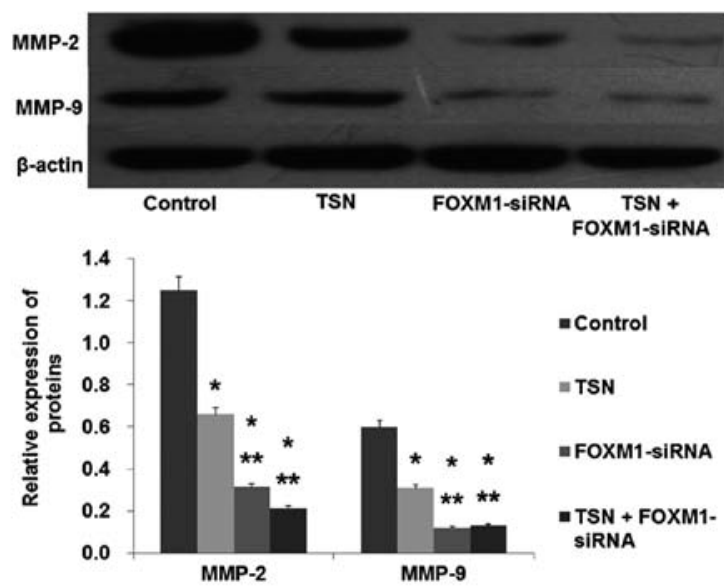

Figure 5. Downregulation of FOXM1 inhibits SGC-7901 cell migration. (A) Wound healing assay showed that downregulation of FOXM1 inhibited SGC-7901 cell migration. (B) Downregulation of FOXM1 decreased the expression levels of MMP-2 and MMP-9; ${ }^{*}$ p $<0.01$ compared with the control; ${ }^{* *}$ p $<0.01$ compared with TSN. TSN, tanshinone IIA.

increased the expression of $\mathrm{P} 21$ and decreased the expression of Ki-67 and PCNA (Fig. 4B; p<0.05). In addition, the migration ability of SGC-7901 cells was also suppressed after being transfected with FOXM1-siRNA (Fig. 5; p<0.05). These data showed that downregulation of FOXM1 had the same effect as TSN on SGC-7901 cell proliferation and migration.

Overexpression of FOXM1 reverses the effect of TSN on $S G C-7901$ cells. To further elucidate the role of FOXM1 in TSN-induced inhibition of SGC-7901 cell proliferation and migration, pcDNA3.1+HA-FOXM1 was transfected into SGC-7901 cells to increase the expression of FOXM1. Fluorescence revealed that the plasmid was successfully transfected into the SGC-7901 cells (Fig. 6A). Western blot analysis demonstrated that the protein level of FOXM1 was increased in the cells transfected with pcDNA3.1+HA-FOXM1 compared with the empty vector (Fig. 6B; p<0.01). Furthermore, quantitative real-time PCR revealed similar results to those obtained from the western blot analysis (Fig. 6C; p<0.01).

After transfection with pcDNA3.1+HA-FOXM1 or the empty plasmid, SGC-7901 cells were incubated with TSN
(5 $\mu \mathrm{g} / \mathrm{l})$ or the vehicle. Compared with the control group, overexpression of FOXM1 increased the proliferation ability of the SGC-7901 cells (Fig. 7A; p<0.01). In addition, we found that overexpression of FOXM1 also decreased the expression of $\mathrm{P} 21$ and increased the expression of PCNA and Ki-67 (Fig. 7B; p<0.01). Moreover, compared with the TSN group, overexpression of FOXM1 not only suppressed the TSN-induced inhibition of SGC-7901 proliferation, but also reversed the TSN-induced decreased expression of PCNA and Ki-67 (Fig. 4; p<0.01). These results suggested that FOXM1 was involved in TSN-induced inhibition of SGC-7901 cell proliferation.

The results from the wound healing assay showed that overexpression of FOXM1 increased the migration ability of the SGC-7901 cells compared with the control group (Fig. 8A; $\mathrm{p}<0.01)$. Moreover, we found that overexpression of FOXM1 also increased the expression of MMP-2 and MMP-9 (Fig. 8B; $\mathrm{p}<0.01)$. In addition, our results revealed that compared with the TSN group, overexpression of FOXM1 suppressed the TSN-induced inhibition of SGC-7901 cell migration, and reversed the TSN-induced decreased expression of MMP-2 


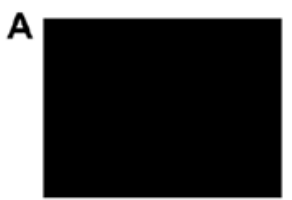

Control

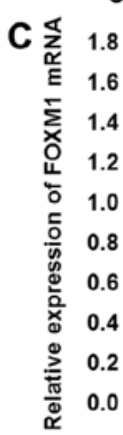

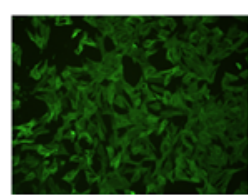

Empty vector

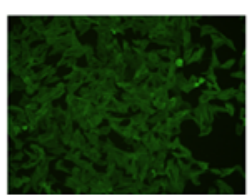

FOXM1
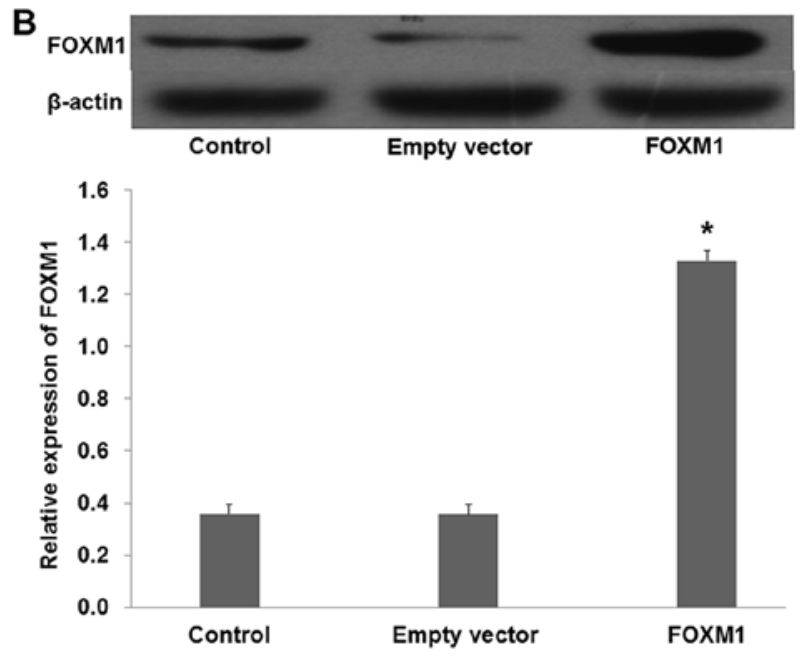

Figure 6. Overexpression of FOXM1 in SGC-7901 cells. (A) Fluorescence showed that the plasmid was successfully transfected into the SGC-7901 cells. (B) Western blot analysis demonstrated that the expression of FOXM1 was increased in the cells transfected with pcDNA3.1+HA-FOXM1. (C) RT-PCR showed that the expression of FOXM1 mRNA was increased in the cells transfected with pcDNA3.1+HA-FOXM1; "p<0.01 compared with the empty vector.
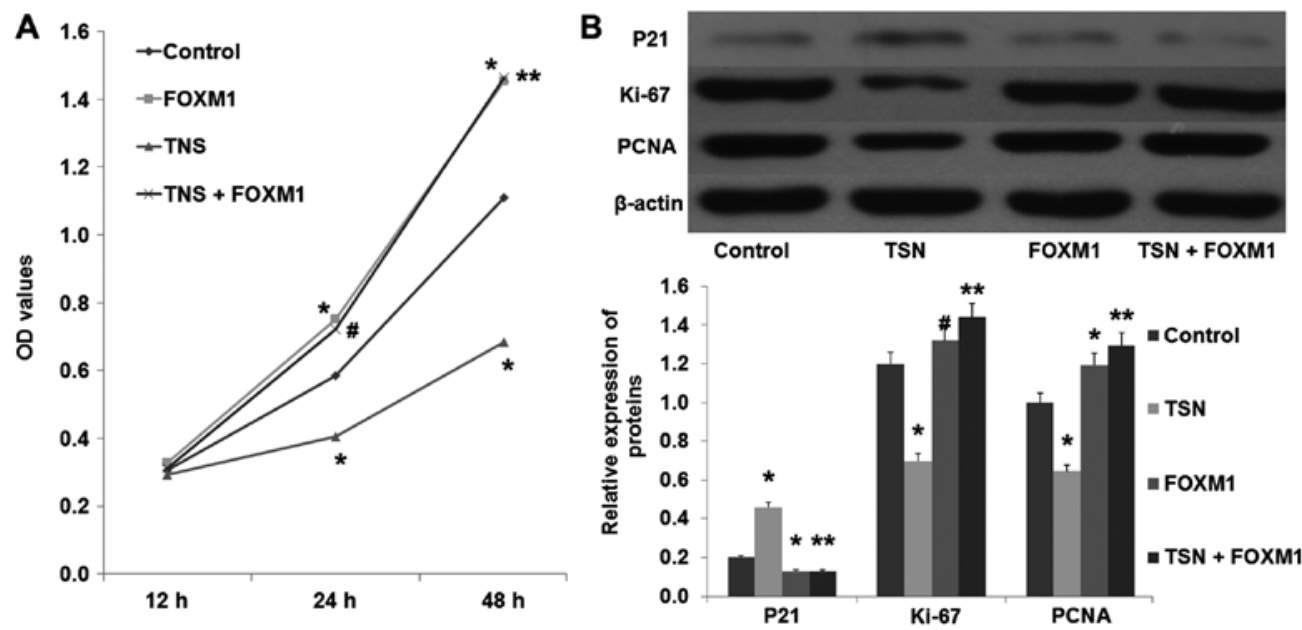

Figure 7. Overexpression of FOXM1 reverses TSN-induced inhibition of SGC-7901 cell proliferation. (A) An MTT assay showed that overexpression of FOXM1 reversed TSN-induced inhibition of SGC-7901 cell proliferation. (B) Overexpression of FOXM1 increased the expression of Ki-67 and PCNA and decreased the expression of P21; ${ }^{*} \mathrm{p}<0.01$ compared with the control; ${ }^{* *} \mathrm{p}<0.01$ compared with TSN. TSN, tanshinone IIA.

\section{A}
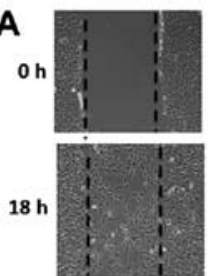

Control

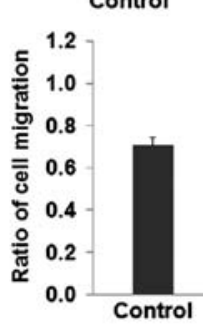

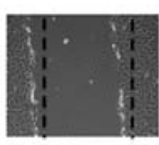

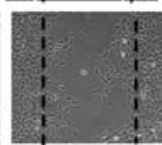

TSN
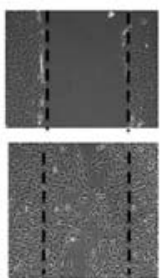

FOXM1
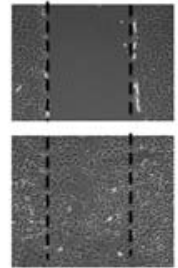

TSN+ FOXM1

\section{$B_{\text {MMP-2 }}$

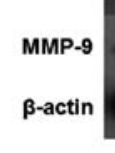

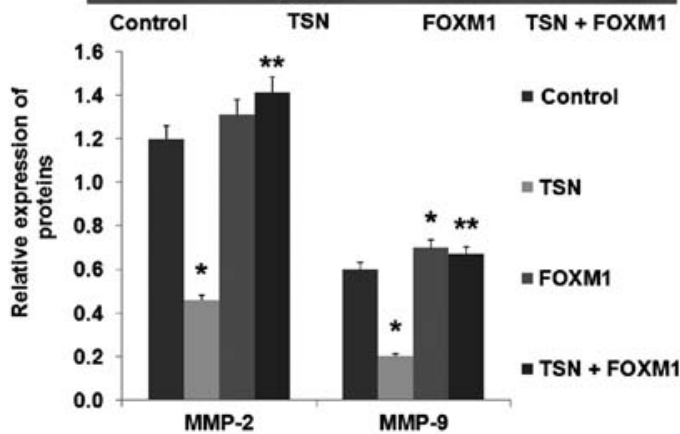

MMP-2

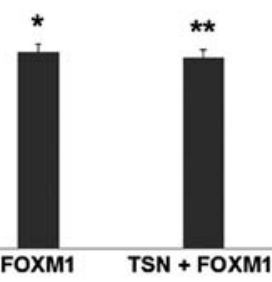

Figure 8. Overexpression of FOXM1 reverses TSN-induced inhibition of SGC-7901 cell migration. (A) Wound healing assay showed that overexpression of FOXM1 reversed TSN-induced inhibition of SGC-7901 cell migration. (B) Overexpression of FOXM1 increased the expression levels of MMP-2 and MMP-9; ${ }^{*} \mathrm{p}<0.01$ compared with the control; ${ }^{* *} \mathrm{p}<0.01$ compared with TSN. TSN, tanshinone IIA. 
and MMP-9 (Fig. 8; p<0.01). These results strongly suggested that FOXM1 was involved in the TSN-induced inhibition of SGC-7901 cell migration.

\section{Discussion}

Abnormal proliferation and migration of tumor cells are crucial pathological processes involved in malignant tumors (13-16). Tumor metastasis is a complex process which includes migratory tumor cells leaving the primary tumor by invasion, disseminating throughout the body via the circulatory system, and colonizing eventually at distant organs (17). It has already been proven that tumor cells acquiring the excessive ability of proliferation and migration and departing from the original locality is a prerequisite for metastasis. Therefore, it is important to find an effective anti-gastric cancer (GC) cell proliferation and migration approach in order to improve the prognosis of patients with GC.

Tanshinone is a herbal medicine derived from the dried root of Salvia miltiorrhiza, which has been widely used in China for hundreds of years. Recently, the pharmacological properties of tanshinone have attracted great interest. Tanshinone IIA (TNS) is the main component of tanshinone, and a variety of clinical trials and experimental studies have demonstrated the protective effect of TSN on cardiovascular diseases, diabetes and tumors (2,18-21). In GC, Jing et al first reported that TSN induced apoptosis and growth inhibition in vitro and in vivo. Subsequent research showed that TSN could reverse the malignant phenotype of GC cells and induce pro-survival autophagy in GC cells $(22,23)$. In accordance with these studies, our research found that TSN inhibited the proliferation and migration ability of SGC-7901 cells in a dose-dependent manner. As a multi-target drug, the molecular targets of TSN include apoptotic-regulating proteins, transcription and growth factors, ion channels and inflammatory mediators $(6,7,24,25)$. In the present study, we found that TSN decreases the expression of FOXM1 in SGC-7901 cells in a dose-dependent manner.

FOXM1 is an important transcription factor required for tissue development and differentiation in vertebrates (26). FOXM1 binds to sequence-specific motifs on DNA (C/TAAACA) through its DNA-binding domain (DBD) and activates proliferation-, migration- and EMT-associated genes. Aberrant overexpression of FOXM1 is a key feature in oncogenesis and progression of many types of human cancer (27). Recently, overexpression of FOXM1 was correlated with the poor prognosis of patients with malignant tumors and has been reported in many types of cancers including GC (28-32). Zhang et al reported that downregulation of FOXM1 suppressed PLK1-regulated cell cycle progression in renal cancer cells (33). Additionally, Inoguchi et al found that microRNA-24-1 inhibited bladder cancer cell proliferation by targeting FOXM1 (34). Therefore, we inferred that TSN inhibited SGC-7901 cell proliferation and migration via the downregulation of FOXM1. Consistent with these studies, our results showed that knockdown of FOXM1 by siRNA had the same effect as TSN on SGC-7901 cells including suppression of cell proliferation and migration, inhibition of the expression of Ki-67, PCNA and MMP-2/-9 and an increase in the expression of P21, which indicated that FOXM1 plays an important role in the regulation of SGC-7901 cell proliferation and migration. Additionally, we also found that overexpression of FOXM1 increases the expression of Ki-67, PCNA, MMP-2/-9 and promotes the proliferation and migration abilities of the SGC-7901 cells. Moreover, our results demonstrated that overexpression of FOXM1 reverses TSN-induced inhibition of SGC-7901 cell proliferation and migration. These results demonstrated that TSN inhibits SGC-7901 cell proliferation and migration via the downregulation of FOXM1.

In summary, the present study provides new insights into the effect of TSN on SGC-7901 cells and the related mechanism. The present study suggests that TSN inhibits proliferation and migration of SGC-7901 cells through, at least in part, the downregulation of FOXM1.

\section{References}

1. Wang T, Chen T, Niu H, Li C, Xu C, Li Y, Huang R, Zhao J and Wu S: MicroRNA-218 inhibits the proliferation and metastasis of esophageal squamous cell carcinoma cells by targeting BMII. Int J Mol Med 36: 93-102, 2015.

2. Xu S, Little PJ, Lan T, Huang Y, Le K, Wu X, Shen X, Huang H, Cai Y, Tang F, et al: Tanshinone II-A attenuates and stabilizes atherosclerotic plaques in apolipoprotein-E knockout mice fed a high cholesterol diet. Arch Biochem Biophys 515: 72-79, 2011.

3. Wang J, Jiang Q, Wan L, Yang K, Zhang Y, Chen Y, Wang E, Lai N, Zhao L, Jiang H, et al: Sodium tanshinone IIA sulfonate inhibits canonical transient receptor potential expression in pulmonary arterial smooth muscle from pulmonary hypertensive rats. Am J Respir Cell Mol Biol 48: 125-134, 2013.

4. Wang X, Wei Y, Yuan S, Liu G, Lu Y, Zhang J, Wang W: Potential anticancer activity of tanshinone IIA against human breast cancer. Int J Cancer 116: 799-807, 2005.

5. Zhang HS, Zhang FJ, Li H, Liu Y, Du GY and Huang YH: Tanshinone IIA inhibits human esophageal cancer cell growth through miR-122-mediated PKM2 down-regulation. Arch Biochem Biophys 598: 50-56, 2016.

6. Qiu S, Granet R, Mbakidi JP, Brégier F, Pouget C, Micallef L, Sothea-Ouk T, Leger DY, Liagre B, Chaleix V, et al: Delivery of tanshinone IIA and $\alpha$-mangostin from gold/PEI/cyclodextrin nanoparticle platform designed for prostate cancer chemotherapy. Bioorg Med Chem Lett 26: 2503-2506, 2016.

7. Lu M, Wang C and Wang J: Tanshinone I induces human colorectal cancer cell apoptosis: The potential roles of Aurora A-p53 and survivin-mediated signaling pathways. Int J Oncol 49: 603-610, 2016.

8. Kim EO, Kang SE, Im CR, Lee JH, Ahn KS, Yang WM, Um JY, Lee SG and Yun M: Tanshinone IIA induces TRAIL sensitization of human lung cancer cells through selective ER stress induction. Int J Oncol 48: 2205-2212, 2016.

9. Sanders DA, Ross-Innes CS, Beraldi D, Carroll JS and Balasubramanian S: Genome-wide mapping of FOXM1 binding reveals co-binding with estrogen receptor alpha in breast cancer cells. Genome Biol 14: R6, 2013.

10. Ahmed M, Hussain AR, Siraj AK, Uddin S, Al-Sanea N, Al-Dayel F, Al-Assiri M, Beg S and Al-Kuraya KS: Co-targeting of Cyclooxygenase-2 and FoxM1 is a viable strategy in inducing anticancer effects in colorectal cancer cells. Mol Cancer 14: 131, 2015.

11. Zhang N, Xie Y, Li B, Ning Z, Wang A and Cui X: FoxM1 influences mouse hepatocellular carcinoma metastasis in vitro. Int J Clin Exp Pathol 8: 2771-2778, 2015.

12. Zhang J, Zhang J, Cui X, Yang Y, Li M, Qu J, Li J and Wang J: FoxM1: A novel tumor biomarker of lung cancer. Int J Clin Exp Med 8: 3136-3140, 2015.

13. Zhang Y, Li CF, Ma LJ, Ding M and Zhang B: MicroRNA-224 aggrevates tumor growth and progression by targeting mTOR in gastric cancer. Int J Oncol 49: 1068-1080, 2016.

14. Kim HY, Cho Y, Kang H, Yim YS, Kim SJ, Song J and Chun KH: Targeting the WEE1 kinase as a molecular targeted therapy for gastric cancer. Oncotarget: Jun 23, 2016 (Epub ahead of print). doi: 10.18632/oncotarget.10231. 
15. Kanda M, Shimizu D, Fujii T, Tanaka H, Tanaka Y, Ezaka K, Shibata M, Takami H, Hashimoto R, Sueoka S, et al: Neurotrophin receptor-interacting melanoma antigen-encoding gene homolog is associated with malignant phenotype of gastric cancer. Ann Surg Oncol 23 (Suppl 4): S532-S539, 2016.

16. Kanda M, Shimizu D, Fujii T, Tanaka H, Shibata M, Iwata N, Hayashi M, Kobayashi D, Tanaka C, Yamada S, et al: Protein arginine methyltransferase 5 is associated with malignant phenotype and peritoneal metastasis in gastric cancer. Int $\mathbf{J}$ Oncol 49: 1195-1202, 2016.

17. Han TS, Hur K, Xu G, Choi B, Okugawa Y, Toiyama Y, Oshima H, Oshima M, Lee HJ, Kim VN, et al: MicroRNA-29c mediates initiation of gastric carcinogenesis by directly targeting ITGB1. Gut 64: 203-214, 2015.

18. Yu ZL, Wang JN, Wu XH, Xie HJ, Han Y, Guan YT, Qin Y and Jiang JM: Tanshinone IIA prevents rat basilar artery smooth muscle cells proliferation by inactivation of PDK1 during the development of hypertension. J Cardiovasc Pharmacol Ther 20 : 563-571, 2015.

19. Wang C, Du X, Yang R, Liu J, Xu D, Shi J, Chen L, Shao R, Fan G, Gao X, et al: The prevention and treatment effects of tanshinone IIA on oestrogen/androgen-induced benign prostatic hyperplasia in rats. J Steroid Biochem Mol Biol 145: 28-37, 2015.

20. Tan X, Yang Y, Cheng J, Li P, Inoue I and Zeng X: Unique action of sodium tanshinone II-A sulfonate (DS-201) on the $\mathrm{Ca}^{2+}$ dependent $\mathrm{BK}_{\mathrm{Ca}}$ activation in mouse cerebral arterial smooth muscle cells. Eur J Pharmacol 656: 27-32, 2011.

21. Yu Q, Sheng L, Yang M,Zhu M,Huang X and Li Q: Tanshinon IIA injection accelerates tissue expansion by reducing the formation of the fibrous capsule. PLoS One 9: e105756, 2014.

22. Jing X, Xu Y, Cheng W, Guo S, Zou Y and He L: Tanshinone I induces apoptosis and pro-survival autophagy in gastric cancers. Cancer Chemother Pharmacol 77: 1171-1181, 2016.

23. Xu M, Cao FL, Li NY, Liu YQ, Li YP and Lv CL: Tanshinone IIA reverses the malignant phenotype of SGC7901 gastric cancer cells. Asian Pac J Cancer Prev 14: 173-177, 2013.

24. Wang Y, Li JX, Wang YQ and Miao ZH: Tanshinone I inhibits tumor angiogenesis by reducing Stat 3 phosphorylation at Tyr705 and hypoxia-induced HIF- $1 \alpha$ accumulation in both endothelial and tumor cells. Oncotarget 6: 16031-16042, 2015.
25. Lin JY, Ke YM, Lai JS, Ho TF: Tanshinone IIA enhances the effects of TRAIL by downregulating survivin in human ovarian carcinoma cells. Phytomedicine 22: 929-938, 2015.

26. Wang CY, Hua L, Sun J, Yao KH, Chen JT, Zhang JJ and Hu JH: MiR-211 inhibits cell proliferation and invasion of gastric cancer by down-regulating SOX4. Int J Clin Exp Pathol 8: 14013-14020, 2015.

27. Gormally MV, Dexheimer TS, Marsico G, Sanders DA, Lowe C, Matak-Vinković D, Michael S, Jadhav A, Rai G, Maloney DJ, et al: Suppression of the FOXM1 transcriptional programme via novel small molecule inhibition. Nat Commun 5: 5165, 2014.

28. Song IS, Jeong YJ, Jeong SH, Heo HJ, Kim HK, Bae KB, Park YH, Kim SU, Kim JM, Kim N, et al: FOXM1-induced PRX3 regulates stemness and survival of colon cancer cells via maintenance of mitochondrial function. Gastroenterology 149: 1006-1016.e9, 2015.

29. Buchner M, Park E, Geng H, Klemm L, Flach J, Passegué E, Schjerven H, Melnick A, Paietta E, Kopanja D, et al: Identification of FOXM1 as a therapeutic target in B-cell lineage acute lymphoblastic leukaemia. Nat Commun 6: 6471, 2015.

30. Wiseman EF, Chen X, Han N, Webber A, Ji Z, Sharrocks AD and Ang YS: Deregulation of the FOXM1 target gene network and its coregulatory partners in oesophageal adenocarcinoma. Mol Cancer 14: 69, 2015.

31. Katoh M and Katoh M: Human FOX gene family (Review). Int J Oncol 25: 1495-1500, 2004.

32. Hui MK, Chan KW, Luk JM, Lee NP, Chung Y, Cheung LC, Srivastava G, Tsao SW, Tang JC and Law S: Cytoplasmic Forkhead box M1 (FoxM1) in esophageal squamous cell carcinoma significantly correlates with pathological disease stage. World J Surg 36: 90-97, 2012.

33. Zhang Z, Zhang G and Kong C: FOXM1 participates in PLK1-regulated cell cycle progression in renal cell cancer cells. Oncol Lett 11: 2685-2691, 2016.

34. Inoguchi S, Seki N, Chiyomaru T, Ishihara T, Matsushita R, Mataki H, Itesako T, Tatarano S, Yoshino H, Goto Y, et al: Tumour-suppressive microRNA-24-1 inhibits cancer cell proliferation through targeting FOXM1 in bladder cancer. FEBS Lett 588: 3170-3179, 2014. 